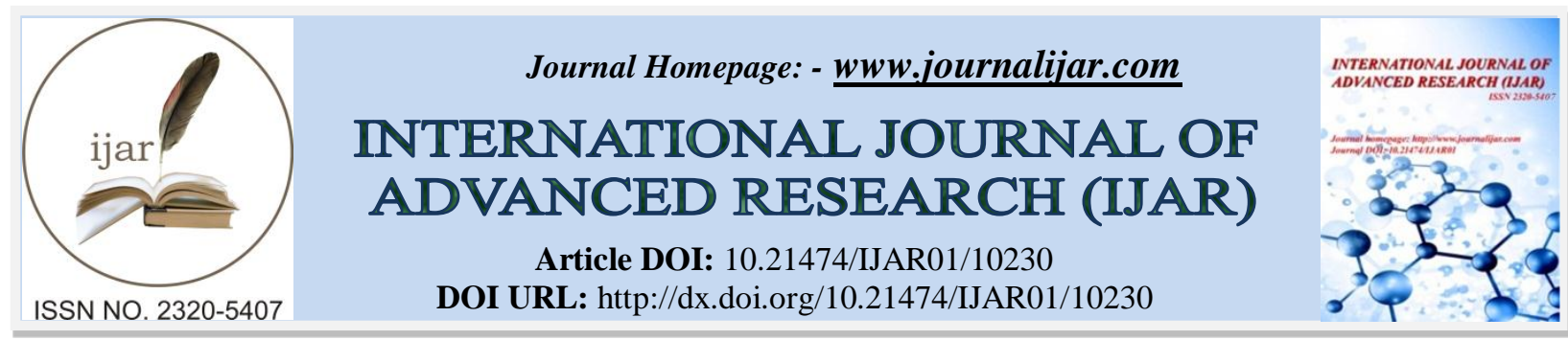

RESEARCH ARTICLE

\title{
SERIAL MONITORING OF SERUM BONE SPECIFIC ALKALINE PHOSPHATASE (BAP) LEVELS FOLLOWING INTRAMEDULLARY FIXATION OF TIBIAL SHAFT FRACTURES
}

\author{
Bansal S. ${ }^{1}$, Dwivedi D.D. ${ }^{2}$, Kumar $\mathrm{S}^{3}$, Puri D. ${ }^{4}$, Mehndiratta M. ${ }^{5}$ and Bansal P $^{6}$ \\ 1. Department of Orthopaedics, 7 Air Force Hospital, Cantonment Area, Kanpur. \\ 2. Senior Resident, MAMC, Delhi. \\ 3. Professor, Department of Orthopedics, Sharda University, Noida. \\ 4. Professor and Head, Department of Biochemistry, UCMS, Delhi. \\ 5. Associate Professor, Department of Biochemistry, UCMS, Delhi. \\ 6. Associate Professor, Department of Biochemistry, BPSGMC, Sonepat.
}

\section{Manuscript Info}

\section{Manuscript History}

Received: 10 October 2019

Final Accepted: 12 November 2019

Published: December 2019

Key words:-

Bone Specific Alkaline Phosphatase, Delayed Union, Tibial Shaft Fracture, RUST Score

Corresponding Author:- Bansal S

Address:- Department of Orthopaedics, 7 Air Force Hospital, Cantonment Area, Kanpur.

\begin{abstract}
Background: Early detection of delayed union or non-union of a fracture is not possible only by clinico-radiological assessment. The changes in the bone turnover markers (BTMs) during fracture healing may lead to early detection of delayed or non-union. Material and methods: Serial measurement of serum Bone Specific Alkaline Phosphatse (BAP) was undertaken after intramedullary fixation of thirty cases (24 males and 6 females) of isolated closed diaphyseal fractures of tibia in healthy young adults (18-45 years age) with 24 weeks follow-up. The fracture was assessed clinically every four weeks and radiologically at 4, 8, 12, 18 and 24 weeks and RUST scoring was done. Serum BAP levels were measured at $0,4,8$ and 12 weeks using commercial ELISA method. Results: 27 (90\%) patients had bridging callus in 3 or 4 cortices (radiological union) and 3 patients (10\%) with delayed union had callus in only two or less cortices at 24 weeks. In patients with normal union peak BAP levels were seen at 8 weeks. BAP levels at 4,8 and 12 weeks were significantly higher in patients achieving normal union as compared to patients with delayed union $(\mathrm{p}=$ $0003,<0.001$ and 0.041 respectively). In delayed union cases though baseline BAP was comparable to that in normal union cases, but it declined afterwards and remained persistently low. RUST score at 12 weeks correlated significantly with BAP level at 8 weeks $(r=0.441$, $\mathrm{p}=0.015)$. RUST score at 18 weeks correlated significantly with BAP at 8 weeks $(r=0.402, p=0.028)$ and $B A P$ at 12 weeks $(r=0.391, p=0.033)$. RUST score at 24 weeks correlated significantly with BAP at 8 weeks $(\mathrm{r}=0.406, \mathrm{p}=0.026)$. Conclusions: Peak BAP levels were seen at 8 weeks and correlated well with RUST scores at 12, 18 and 24 weeks. Thus a rising BAP level up to 8 weeks may predict normal radiological healing in coming weeks. While a failure of BAP to rise and persistent low BAP levels than baseline up to 8 weeks may be predictive of delayed union, and a larger study is recommended for better analysis.
\end{abstract}




\section{Introduction:-}

The detection of bridging callus in three or more cortices across the fracture site in combination with clinical signs of union remain the gold standard for assessing fracture union (Leow et al., 2016). Quantification of radiological callus can be assessed by the radiographic union score for tibial fractures (RUST) score (Leow et al., 2016). But early detection of delayed or non-healing fracture is not possible using these methods only. The changes in the bone turnover markers (BTMs) during fracture repair may lead to early detection of delayed or non union.

Bone turnover markers: The balance in the process of bone formation and resorption determines skeletal mass (Olsen et al., 2000). As the skeleton grows bone formation predominates. Once the skeleton has reached maturity, the breakdown and renewal of bone that is responsible for skeletal maintenance is called remodeling and is likely initiated at sites experiencing fatigue and microdamage (Watts, 1999). This bone turnover can be demonstrated quantitatively by measuring the presence of a number of molecular markers which suggest the amount of formation (osteoblastic activity) or resorption (osteoclastic activity) of bone which is occurring. These 'bone turnover markers' (BTMs) are commonly subdivided into three categories: 1) bone-resorption markers, 2) osteoclast regulatory proteins and 3) bone formation markers (Cox et al., 2010).

Bone-specific alkaline phosphatase (BAP): Bone-specific alkaline phosphatase (BAP) is an isoenzyme produced by osteoblasts involved with calcification of skeleton and bone formation. Bone-specific alkaline phosphatase (BAP) is a specific marker of osteoblasts and gives a good measure of bone forming activity (Coxet al., 2010; Gothlin and Ericsson, 1973). Various studies on healing of long bone fractures describe peak BAP levels by 7 to 14 weeks after treatment initiation followed by return to baseline by 24th week (Leung et al., 1993; Hermann et al.,2002; Bowles et al.,1996; Enami et al., 1999). Enami et al. reported that, patients of long bone fracture with normal healing had significantly higher levels of BAP at 4 weeks than did patients with delayed healing but the difference was not significant at 7 weeks (Enami et al., 1999). While Hermann et al. reported that in patients of long bone fracture with delayed healing, BAP at baseline was significantly higher and tended to increase throughout the study period (Hermann et al.,2002). Kurdy studied 20 patients of which the three fractures exhibiting delayed union demonstrated a persistent decline in BAP from 10 weeks onward (Kurdy,2000). Thus while serial monitoring of BAP up to 12 weeks has potential to aid in early diagnosis and prediction of delayed or non union conflicting reports exist in literature regarding levels and status of BAP. Bone turnover markers have been studied in various fractures, however fractures in tibia are a good model to study these markers due to common frequency of these fractures, amount of bone turnover markers produced and incidence of delayed union (Cox et al., 2010). Considering all of above, this study was thus designed to assess the correlation between serial levels of BAP and radiological RUST score and to compare the serial levels of BAP and clinical and radiological signs of fracture.

\section{Patients and Methods: - Study design:}

A prospective, non-randomised, observational study to evaluate the serial changes in serum bone-specific alkaline phosphatase levels during healing of isolated tibial shaft fractures was conducted in Department of Orthopaedics and Biochemistry, University College of Medical Sciences, Delhi. 30 otherwise healthy adults in the age group 18-45 years with closed fracture shaft of tibia were included. Patients with any liver disease, deranged liver function tests, systemic disease, and/or drug intake affecting bone turnover in any manner and post menopausal females were excluded from the study. Approval of Institutional ethical committee was taken and informed voluntary consent was taken from all patients recruited in the study. All these fractures underwent closed reduction and internal fixation with intramedullary interlocking nail and were followed up for 24 weeks.

\section{Clinical and radiological assessment:}

The fracture healing was assessed clinically by local tenderness and ability to bear weight on the affected limb with or without support every four weeks. Radiological assessment of fracture healing was done at 4, 8, 12, 18 and 24 weeks. Patients with bridging callus (fracture line not visible) in three or four cortices in anteroposterior and lateral radiograph of fracture site were labelled as united (Gothlin and Ericsson, 1973). Patients having bridging callus in less than three cortices at the end of 24 weeks were labelled as cases of delayed union (Whelan et al., 2002). The radiological quantification of callus formation was done using standard antero- posterior and lateral views. When no callus was present the RUST score was one for one cortex. When callus was present but fracture line was visible the 
score was two for one cortex. When callus was present and fracture line was not visible score was 3 for one cortex. Hence in fully consolidated bone the score was 12. The score of twelve indicated excellent callus formation (Leow et al., 2016).

\section{BAP measurement:}

Venous sample were obtained on the day of presentation (baseline), 4 weeks, 8 weeks and 12 weeks post injury. The serum was stored at $-40^{\circ} \mathrm{C}$. Samples were analyzed for BAP by commercial enzyme immunoassay using method as

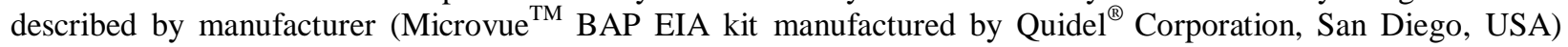
(Gomez et al., 1995). Appropriate quality controls were also used to ensure accuracy of BAP measurement. Results were documented on each patient sheet and registered in a database. Reference range of BAP in adult males is 15.041.3 U/L and in adult premenopausal females is 11.6-29.6 U/L as per the kit used.

\section{Data analysis:-}

The elevation in the mean levels of BAP from baseline at 4, 8 and 12 weeks was compared using Wilcoxon Signed Ranks Test. The BAP levels in different patients were compared using Mann Whitney test. Correlations between BAP and RUST score were assessed by Spearman correlation test. SPSS ver.21 was used for statistical analysis and graph preparation.

\section{Results:-}

General characteristics, Clinical and radiological assessment: Of the 30 patients included from age range of 18 - 45 years, 24 patients were male and 6 were female. 50\% (15 patients) had road traffic accident, $43.3 \%$ (13 patients) had household injury, while 6.6\% (2 patients) fell from height. $60 \%$ patients had high energy trauma while $40 \%$ had low energy trauma. $73.3 \%$ (22 patients) had fracture level at middle third of tibial shaft, $20 \%$ (6 patients) had fracture in lower third and rest $6.7 \%$ (2 patients) had fracture in upper third of tibia. $73.3 \%$ (22 patients) had non-comminuted fracture while rest $26.7 \%$ (8 patients) had comminuted fracture. $90 \%$ (27 patients) had associated fibular fracture while in 10\% (3 patients) fibula was intact. 46.6\% (14 patients) had transverse fracture, $40 \%$ (12 patients) had oblique fracture and $13.3 \%$ (4patients) had spiral type of fracture. Serum calcium and phosphorus levels were within reference range in all patients at all times of follow-up. Percentage of patients with tenderness, weight bearing without support at each follow up is summarized in table 1. Radiological findings (no. of cortices with bridging callus) are summarized in table 2. At 24 weeks 3 patients had bridging callus at only two or less cortices, these patients were labelled as cases of delayed union.

Serial BAP levels and correlations: Mean BAP levels and RUST score in patients with optimal and delayed union are described in table-3.

Table 1:- Clinical features at different time of follow up.

\begin{tabular}{|l|l|l|}
\hline & Tenderness & Weight bearing without support \\
\hline 4 weeks & $30(100 \%)$ & 0 \\
\hline 8 weeks & $15(50 \%)$ & $12(40 \%)$ \\
\hline 12 weeks & $3(10 \%)$ & $25(83.3 \%)$ \\
\hline 18 weeks & $3(10 \%)$ & $27(90 \%)$ \\
\hline 24 weeks & $1(3.3 \%)$ & $27(90 \%)$ \\
\hline
\end{tabular}

Table 2:- Radiological features at different times of follow up.

\begin{tabular}{|l|l|l|l|}
\hline & $\begin{array}{l}\text { Bridging callus in 1 cortex, } \\
\text { with fracture line not } \\
\text { visible }\end{array}$ & $\begin{array}{l}\text { Bridging callus in 2 } \\
\text { cortices, with fracture line } \\
\text { not visible }\end{array}$ & $\begin{array}{l}\text { Bridging callus in 3 or } 4 \text { cortices, } \\
\text { with fracture line not visible } \\
\text { (Radiological union) }\end{array}$ \\
\hline 8 weeks & $8(26.7 \%)$ & 0 & 0 \\
\hline 12 weeks & $15(50 \%)$ & $11(36.6 \%)$ & 0 \\
\hline 18 weeks & $1(3.3 \%)$ & $5(16.6 \%)$ & $23(76.6 \%)$ \\
\hline 24 weeks & $1(3.3 \%)$ & $2(6.7 \%)$ & $27(90 \%)$ \\
\hline
\end{tabular}

Table 3:- RUST score and BAP levels in patients.

\begin{tabular}{|l|l|l|l|l|}
\hline & \multicolumn{2}{|l|}{ Normal Union by 24 weeks $(\mathrm{n}=27)$} & \multicolumn{2}{l|}{ Delayed union at 24 weeks $(\mathrm{n}=3)$} \\
\hline & Mean RUST score & Mean BAP $(\mathrm{U} / \mathrm{L})$ & Mean RUST score & Mean BAP $(\mathrm{U} / \mathrm{L})$ \\
\hline 0 weeks & - & $17.3 \pm 10.4$ & - & $20.8 \pm 1.3$ \\
\hline
\end{tabular}




\begin{tabular}{|l|l|l|l|l|}
\hline 4 weeks & 4 & $27.6 \pm 12.7$ & 4 & $10.6 \pm 1.5$ \\
\hline 8 weeks & $4.5 \pm 1.0$ & $31.7 \pm 13.4$ & 4 & $13.3 \pm 0.3$ \\
\hline 12 weeks & $8.0 \pm 1.0$ & $26.8 \pm 8.8$ & 4 & $16.9 \pm 3.1$ \\
\hline 18 weeks & $10.0 \pm 1.2$ & - & $5.3 \pm 1.2$ & - \\
\hline 24 weeks & $11.52 \pm 0.5$ & - & $7.7 \pm 0.6$ & - \\
\hline
\end{tabular}

In patients with normal union by 24 weeks, peak BAP levels were seen at 8 weeks (table 3 , figure $1 \& 2$ ). Levels at 4 , 8 and 12 weeks were significantly higher than baseline levels $(\mathrm{p}<0.001)$. BAP levels at 4,8 and 12 weeks were significantly higher in patients achieving normal union as compared to patients with delayed union (table 3 , $\mathrm{p}=$ 0003, <0.001 and 0.041 respectively). In delayed union cases though baseline BAP was comparable to that in normal union cases $(\mathrm{p}=0.224)$, but it declined afterwards and remained persistently low (table 3 , figure 3 ).

By 18 weeks $76.6 \%$ patients had achieved radiological union. RUST score at 12 weeks correlated significantly with BAP level at 8 weeks $(\mathrm{r}=0.441, \mathrm{p}=0.015$, figure 4$)$. RUST score at 18 weeks correlated significantly with BAP at 8 weeks $(r=0.402, p=0.028$, figure 5) and BAP at 12 weeks $(r=0.391, p=0.033)$. RUST score at 24 weeks correlated significantly with BAP at 8 weeks $(\mathrm{r}=0.406, \mathrm{p}=0.026)$. Thus a rising BAP level up to 8 weeks may predict normal radiological healing in coming weeks and a failure of BAP to rise with persistent low BAP levels than baseline may be predictive of delayed union.

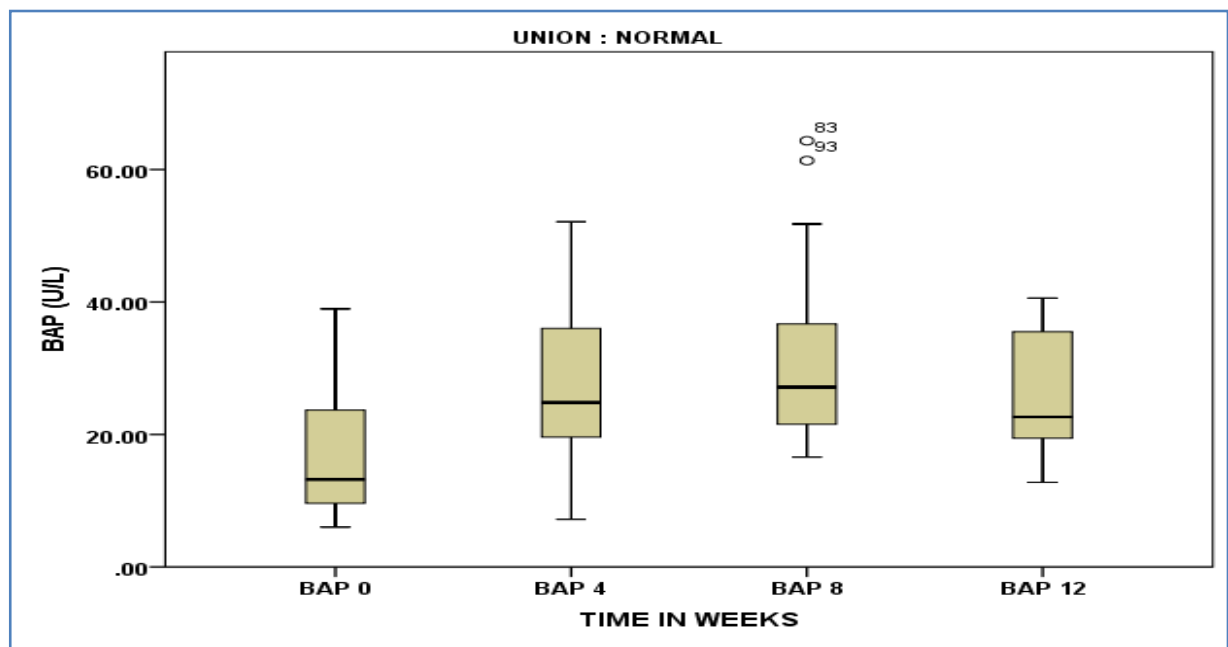

Figure 1:- BAP levels in patients with normal union at $0,4,8$ and 12 weeks.

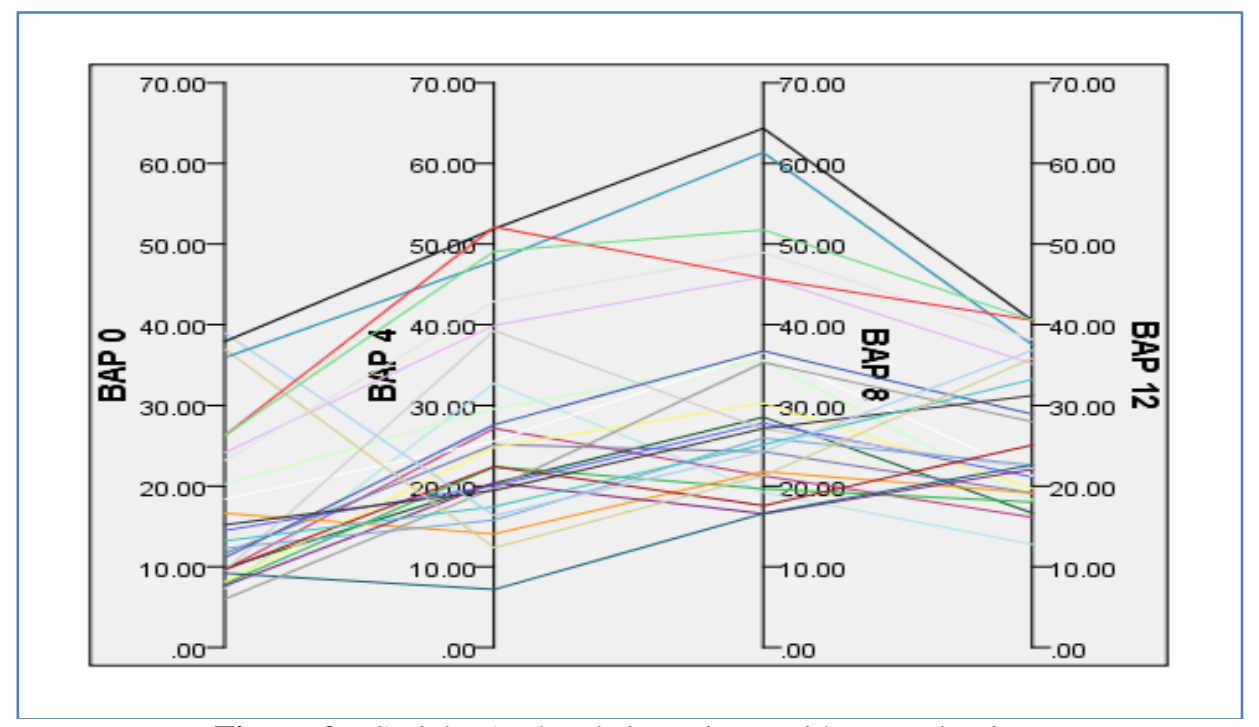

Figure 2:- Serial BAP levels in patients with normal union. 


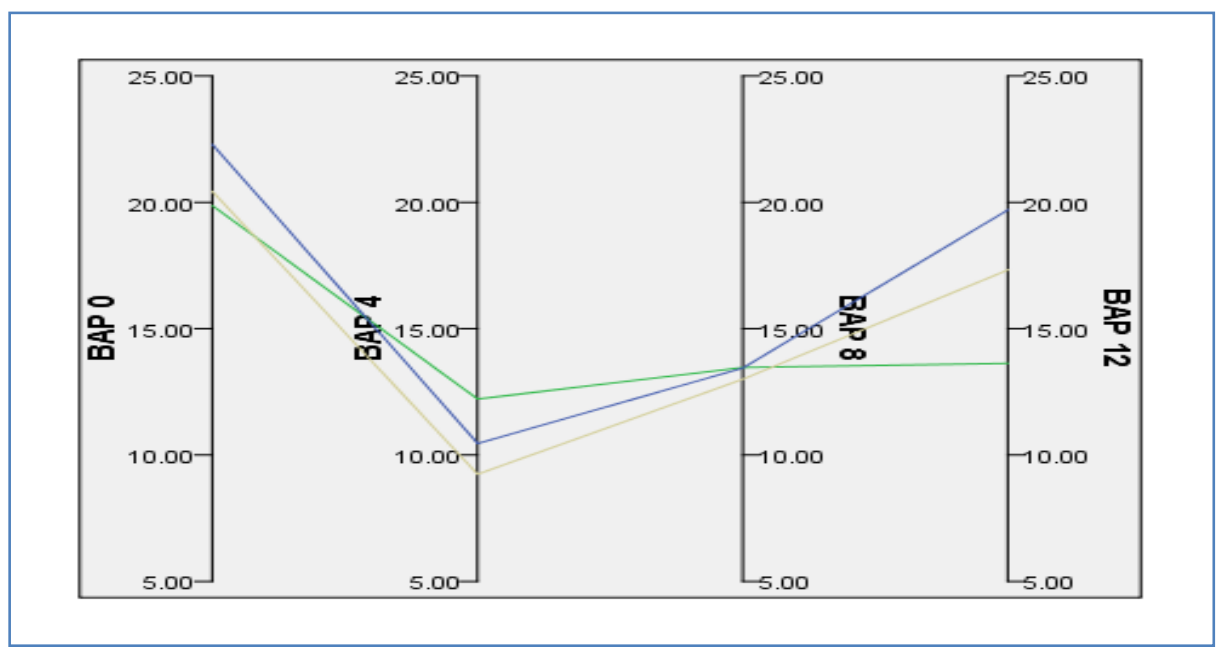

Figure 3:- Serial BAP levels in patients with delayed union.

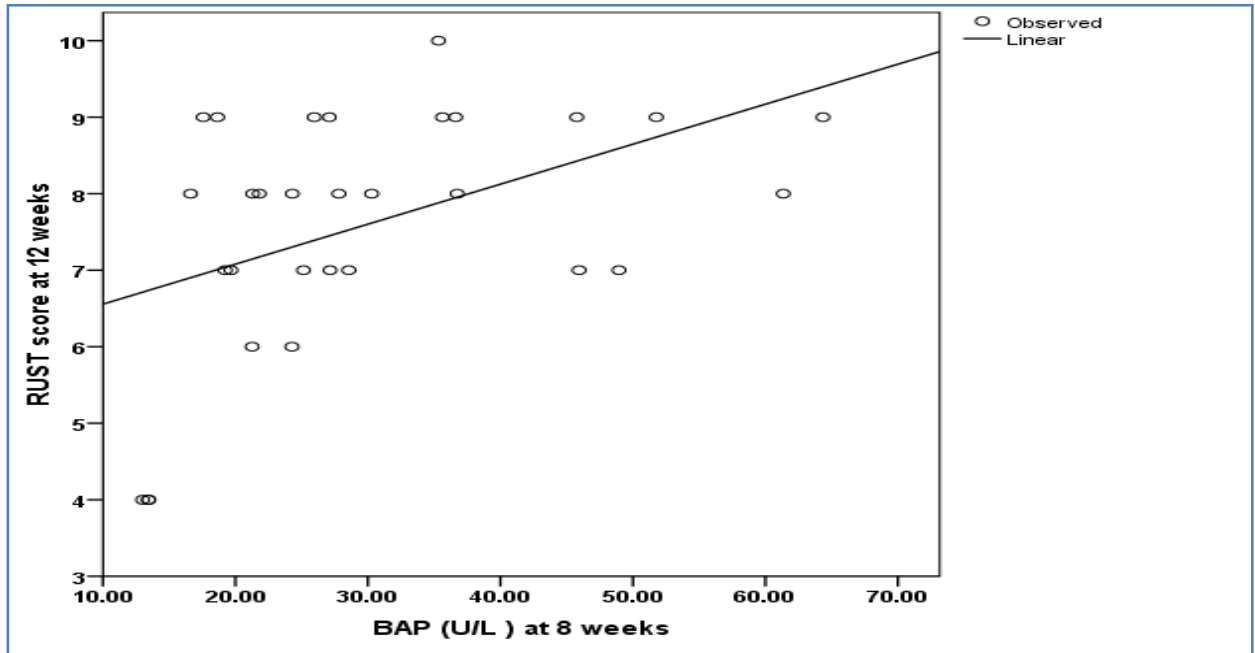

Figure 4:- Correlation of RUST score at 12 weeks with BAP levels at 8 weeks.

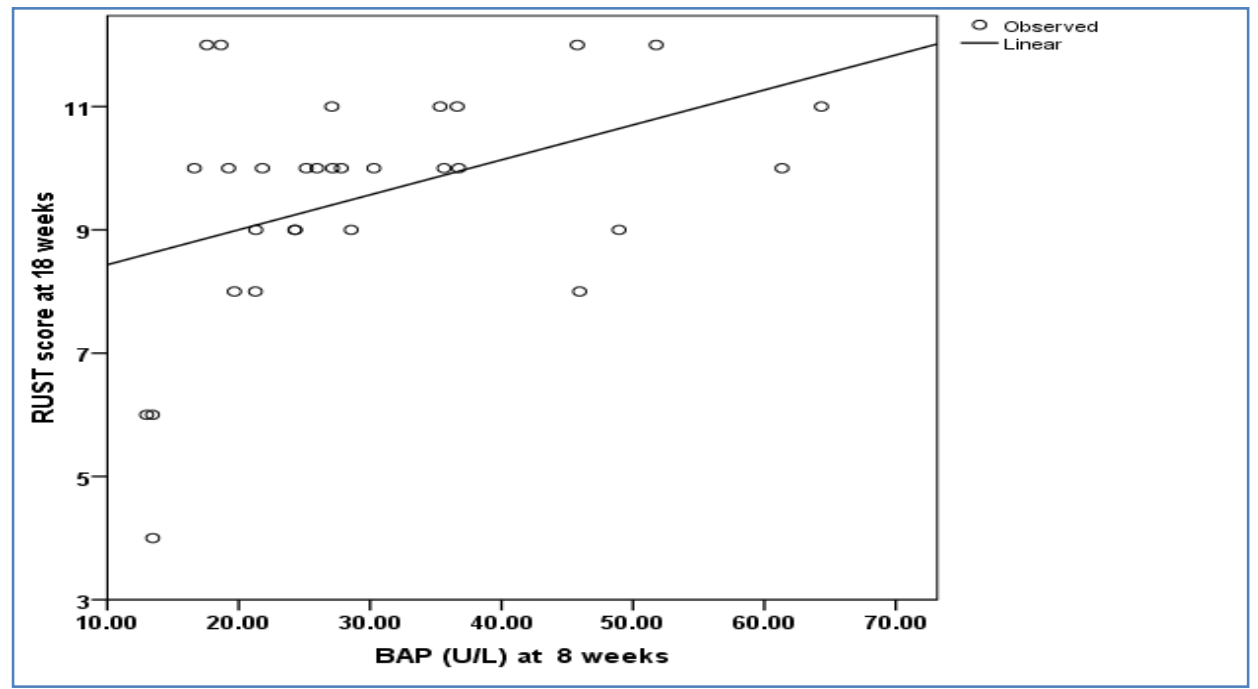

Figure 5:- Correlation of RUST score at 18 weeks with BAP levels at 8 weeks. 


\section{Discussion:-}

Bone specific isoenzyme of Alkaline phosphatase is a marker elevated in all mineralizing tissues. It is an ectoenzyme found in the plasma membrane of osteoblasts and is believed to be having a role in the mineralization of the newly formed bone. BAP is found in the plasma membrane of osteoblasts (Gothlin and Ericsson, 1973). A crossreactivity of approximately $15 \%$ between liver and bone ALP can affect measurements in individuals with high liver ALP (Granchi et al., 2017). Patients with liver disease or deranged liver function tests were excluded from the study. BAP levels are also affected by gender, age and vitamin D status (Leung et al., 1993). Thus change in BAP levels from baseline at 4, 8 and 12 weeks was studied and focussed on in the present study. Fractures in tibia are a good model to study bone turnover marker like BAP due to common frequency of these fractures, larger amount of bone turnover markers produced, incidence of delayed union and use of RUST scoring for assessing the healing and callus formation (Cox et al., 2010). RUST scoring system is a reliable and repeatable outcome measure for assessing tibial fracture healing (Leow et al., 2016). RUST scores of patients correlated well with the clinical assessment of healing in terms of weight bearing and absence of tenderness in the patients included in this study.

The rise in levels of the BAP with peak at 8 weeks in our study was in agreement with studies by Leung et al. (study on 49 long bone fracture adults) and Ingle et al. (study on 14 tibial shaft fracture patients) (Leung et al., 1993; Ingle et al., 1999). Levels were significantly higher at 4, 8 and 12 weeks in cases with normal union than in cases with delayed union (table 3). Enami et al. describing their study in 30 long bone fracture patients reported that patients with normal healing had significantly higher levels of BAP at 4 weeks than did patients with delayed healing (7 cases) but the difference was not significant at 7 weeks (Enami et al., 1999). In the present study, in the three cases with delayed union BAP levels decreased after baseline and remained low (table 3). Kurdy et al. also studied 20 patients of which the three fractures exhibiting delayed union demonstrated a persistent decline in B AP from 10 weeks onward (Kurdy et al., 2000). However, in the study on 14 long bone fracture cases by Hermann et al., in patients with delayed healing (4 cases), BAP at baseline was significantly higher and tended to increase throughout the study period (Hermann et al., 2002). Granchi et al. measured BAP levels after regenerative treatment with autologous expanded Mesenchymal Stem Cells combined with a biphasic calcium phosphate in patients with delayed union or non-union of long bone fractures. They found that BAP peaked between 6 to 12 weeks after surgery and correlated to the imaging results at 12 and 24 weeks. Levels reached baseline by 24 weeks. Further a remarkable decrease in BAP was observed at all time points in a single patient who experienced atreatment failure (Granchi et al., 2017).

In our study, three cases were labeled as delayed union because of presence of bridging callus in only two cortices or less at the end of 24 weeks observation period. Of these, one of the patients was a chronic smoker whereas the other patient, a female, was having a comminuted fracture. These are considered to be risk factors for delayed union (Kyro et al., 1993). Multiple factors have been shown to contribute to delayed union and non-union of tibial fractures. One of them is malnutrition which often goes unrecognised. Adequate protein is required for healing and inadequate caloric intake has been shown to contribute to delayed and non-union (Smith, 1987). Subclinical malnutrition might have been a contributory factor in these cases.

Bone specific alkaline phosphatase is a marker of bone formation and the significantly raised levels of bone specific alkaline phosphatase at 4,8 and 12 weeks after fracture signify the increased osteoblastic activity occurring at the fracture site. Correlation between RUST scores at 12, 18 and 24 weeks with the levels of BAP at 8 weeks (figures 4 $\& 5)$ signify its role in the mineralization of the callus and consolidation of the fracture. Peak BAP levels at 8 weeks correlate well with adequate development of callus in the next few weeks. Serum bone specific alkaline phosphatase at 8 weeks correlates well with the clinico-radiological signs of fracture healing in tibial diaphyseal fracture at 12,18 and 24 weeks.

A fall in the levels of BAP as compared to baseline is one feature which should be monitored closely. The bone turnover markers are suggested as a non-invasive aid for monitoring fracture healing. They may help in the monitoring of fracture healing, complementing the clinico-radiological evaluation. The drawbacks in the above study are small number of patients and only one bone turnover marker estimation. Diagnostic delay in case of a nonunion is costly from both the patient and treatment standpoints. The complex mechanisms and the mysterious pathways of a bone not healing normally need to be revealed as the secret of predicting which injured bone will not heal lies within them. Therefore, sustained efforts need to be continued in our endeavour to find bone turnover markers as diagnostic or prognostic tools for monitoring bone healing. Further studies in this direction can throw more light on the complex process of bone healing and the delicate balance between bone formation and resorption. 


\section{Conclusions:-}

Peak BAP levels were seen at 8 weeks and correlated well with RUST scores at 12,18 and 24 weeks. Thus a rising BAP level up to 8 weeks may predict normal radiological healing in coming weeks. While a failure of BAP to rise and persistent low BAP levels than baseline may be predictive of delayed union, and a larger study is recommended for better analysis.

\section{Declaration of Interest:}

None

\section{Conflict of interest:}

None

\section{Financial acknowledgements:}

None

\section{References:-}

1. Bowles SA, Kurdy N, Davis AM, France MW, Marsh DR. (1996): Serum osteocalcin, total and bone-specific alkaline phosphatase following isolated tibial shaft fracture. Ann. Clin. Biochem., 33:196-200.

2. Cox G, Einhorn TA, Tzioupis C, Giannoudis PV. (2010): Bone-turnover markers in fracture healing. J. Bone Joint Surg., 92-B:329-34.

3. Emami A, Larsson A, Petren-Mallmin M, Larsson S. (1999): Serum bone markersafter intramedullary fixed tibial fractures. Clinic. Orthop., 368:220-9.

4. Gomez B, S Ardakani, J Ju, D Jenkins, M J Cerelli, G Y Daniloff, V T Kung. (1995): Monoclonal antibody assay for measuring bone-specific alkaline phosphatase activity in serum. Clinical Chemistry., 41(11):1560-6.

5. Gothlin G, Ericsson JL. (1973): Fine structural localization of alkaline phosphatase in the fracture callus of the rat. Histochemie., 36:225-36.

6. Granchi D, Gómez-Barrena E, Rojewski M, Rosset P, Layrolle P, Spazzoli B, et al. (2017): Changes of Bone Turnover Markers in Long Bone Nonunions Treated with a Regenerative Approach. Stem Cells Int., 3674045.

7. Herrmann JM, Klitscher D, Georg T, Frank J, Marzi I, Herrmann W. (2002): Different kinetics of bone markers in normal and delayed fracture healing of long bones. Clin. Chem., 48:2263-6.

8. Ingle BM, Hay SM, Bottjer HM, Eastell R. (1999): Changes in bone mass and bone turnover following ankle fracture. Osteoporos. Int., 10:408-15.

9. Kurdy NM. (2000): Serology of abnormal fracture healing: the role of PIINP, PICP and BsALP. J. Orthop. Trauma., 14:48-53.

10. Kyro A, Usenius JP, Aarnio M, Kunnamo I, Avikainen V. (1993): Are smokers a risk group for delayed healing of tibial shaft fractures? Ann. Chir. Gynaecol., 82(4):254-62.

11. Leow JM, Clement ND, Tawonsawatruk T, Simpson CJ, Simpson AH. (2016): The radiographic union scale in tibial (RUST) fractures: Reliability of the outcome measure at an independent centre. Bone Joint Res., 5: 11621.

12. Leung KS, Fung KP, Sher AHL, Li CK, Lee KM. (1993): Plasma bone-specific alkaline phosphatase as an indicator of osteoblastic activity. J. Bone Joint Surg., 75-B:288-92.

13. Olsen BR, Reginato AM, Wang W (2000): Bone development. Annu. Rev. Cell Dev. Biol., 16:191.

14. Smith TK. (1987): Prevention of Complications in Orthopedic Surgery Secondary to Nutritional Depletion. Clin. Orthop. Relat. Res., 222:91-7.

15. Watts NB. (1999): Clinical utility of biochemical markers of bone remodelling. Clin. Chem.,45(8):1359-68.

16. Whelan DB, Bhandari M, McKee MD, Guyatt GH, Kreder HJ, Stephen D, et al. (2002): Interobserver and intraobserver variation in the assessment of the healing of tibial fractures after intramedullary fixation. J. Bone Joint Surg. Br., 84-B: 15-8. 